\title{
DETECTION AND MOLECULAR CHARACTERISATION OF EHRLICHIA CANIS IN NATURALLY INFECTED DOGS IN SOUTH WEST NIGERIA
}

\author{
Olukayode Olugbenga DARAMOLA ${ }^{1}$, Michael Irewole TAKEET ${ }^{2 *}$, Ibironke Kofoworola \\ OYEWUSI $^{1}$, Mufutau Atanda OYEKUNLE ${ }^{2}$ and Adewale Oladele TALABI ${ }^{1}$ \\ ${ }^{1}$ Department of Veterinary Medicine and Surgery and ${ }^{2}$ Department of Veterinary \\ Microbiology and Parasitology, College of Veterinary Medicine, Federal University of \\ Agriculture Abeokuta, Nigeria
}

(Received 10 August 2017; accepted 6 November 2017)

\begin{abstract}
Canine ehrlichiosis is an important tick-borne rickettsial disease mainly caused by Ehrlichia canis. This study aimed to detect and characterise E. canis in dogs in Abeokuta, Nigeria by microscopy and nested PCR. Blood samples were collected from 205 dogs, thin smears were made, field-stained, and DNA was extracted from the blood samples. A partial region of the 16S rRNA gene was amplified by polymerase chain reaction (PCR) and sequenced unidirectionally. Ehrlichial morulae were detected in three dogs $(1.5 \%)$. The PCR test revealed that 47 dogs $(22.9 \%)$ were positive for E. canis. The lengths of the sequences obtained range from 374 bp to 376 bp with an average G-C content of $37 \%$ and $98-99 \%$ homology with the reference sequences in GenBank. The aligned autochthonous sequences were less polymorphic. The phylogenetic analysis separated sequences reported previously in Nigeria from the autochthonous sequences. The present work shows that the strain of E. canis detected in the study area is genetically different from those reported in the northern part of Nigeria and more closely related to sequences from Brazil and India.
\end{abstract} prevalence

Key words: Ehrlichia canis, dog, nested PCR, Abeokuta, 16s rRNA,

Canine ehrlichiosis is a rickettsial bacteria disease of dogs that has worldwide distribution (Engvall et al., 1996; McBride et al., 1996; Souza et al., 2010; Fourie et al., 2013; Milanjeet et al., 2014; Cardoso et al., 2016; Cicuttin et al., 2016; Kaewmongkol et al., 2016). Two forms of the disease are known in dogs and humans. In dogs, these include the monocytic form which is caused by Ehrlichia canis (E. canis) and the granulocytic form that is caused by E. ewingii. They are transmitted by the brown dog tick (Rhipicephalus sanguineus) and the lone star tick called Amblyomma americanum, respectively. In humans, the monocytic and granulocytic forms are caused by E. chaffeensis and E. ewingii, re-

\footnotetext{
*Corresponding author; E-mail: takeetmi@funaab.edu.ng; Phone: 0023 (480) 3787-2682
} 
spectively, though Anaplasma phagocytophilum (formerly E. equi) and E. canis have also been reported in human ehrlichiosis (Dumler et al., 2005; Perez et al., 2006).

The clinical manifestation of canine ehrlichiosis varies depending on the immune status, the breeds of dogs infected and the strain of infecting Ehrlichia species. While E. canis is generally believed to cause more severe febrile condition in dogs, E. ewingii infection is less severe (Liddell et al., 2003). Signs of the disease in dogs may include but are not limited to fever, anaemia, haemorrhages, oedema, lymphadenopathy, thrombocytopenia, anorexia and weight loss (Cicuttin et al., 2016; Lauzi et al., 2016). The organism is characterised by the presence of intracytoplasmic inclusion bodies (morulae) in the monocytes/granulocytes of infected dogs, which can be detected by microscopic examination of peripheral blood (Dagnone et al., 2009). Other techniques such as serology can be used, though with its own limitation to differentiate between previous and ongoing infection of dogs (Cicuttin et al., 2016). In recent years, polymerase chain reaction (PCR) has been found to be more sensitive in the detection of Ehrlichia DNA in the blood of infected animals.

The prevalence and molecular characteristics of Ehrlichia canis have been studied extensively around the world (Suksawat et al., 2001; Unver et al., 2001; Vinasco et al., 2007; Aguiar et al., 2008; Harrus et al., 2011; Nazari et al., 2013; Milanjeet et al., 2014; Cardoso et al., 2016). One of these studies has revealed that more than one strain may exist in a region (Vinasco et al., 2007). In Nigeria, Kamani et al. (2013) shed light on the molecular characteristics of E. canis detected in three northern states of the country. Hence, this study characterised $E$. canis detected in naturally infected dogs in the western part of Nigeria and compared their sequences with those detected elsewhere in the world and those reported by Kamani et al. (2013) in Nigeria.

\section{Materials and methods}

\section{Study area and sampled population}

The study was carried out in Abeokuta metropolis, Ogun State, Nigeria. Ogun state is bounded by Lagos State to the south, Oyo and Osun States to the north, Ondo State to the east and the Republic of Benin to the west. A total of 205 dogs consisting local and exotic breeds were randomly sampled mainly from the dogs presented to the Veterinary Teaching Hospital, Federal University of Agriculture, Abeokuta and various veterinary hospitals in the city. The dogs were grouped to young ( $\leq 1$ year) and adult $(\geq 1$ year) and their sexes were also recorded. The dogs sampled were predominantly exotic breeds, which included Alsatian (102), Rottweiler (18), Boeboel (44), unidentified breed (10) and mongrel (31). Pregnant dogs and those with a history of recent medication (such as 
antibiotic therapy and anti-protozoan therapy) within the last four weeks were excluded from the study.

A blood sample was collected from each dog using cephalic venipuncture into tubes containing ethylenediamine tetraacetic acid (EDTA) as anticoagulant. The collected blood samples were transported on ice packs to the laboratory. Thin blood smears were made from each sample collected as well as smears of the buffy coat from the spun capillary tubes, fixed with $98 \%$ methanol (Dudal diesel, Madison, USA) and then reverse stained with Field stain A and B (Biolab Diagnostics, USA). The slides were examined under an oil immersion objective of a light microscope (Olympus CX21, China). Sample in which intracytoplasmic inclusion bodies were demonstrated were adjudged positive for Ehrlichia spp. (Engvall et al., 1996). Blood samples not processed immediately for DNA extraction were stored at $-20^{\circ} \mathrm{C}$ until use.

\section{DNA extraction and PCR assay for E. canis}

Genomic DNA was extracted from whole blood using a commercially available DNA extraction kit (Quick-gDNA MicroPrep, Zymo Research Corporation, Irvine, USA) following the manufacturer's protocol, and the eluted DNA was stored at $-20^{\circ} \mathrm{C}$ until use as previously described (Takeet et al., 2013). Nested PCR that targeted partial regions of the 16S rRNA gene was carried out using the primers listed (Table 1). The primary and the nested reactions were carried out using primer sets ECC \& ECB and ECAN5 \& HE3 (Table 1), respectively. The primary reaction primers target $478 \mathrm{bp}$ of Ehrlichia spp. while the nested reaction primers are specific for the amplification of $398 \mathrm{bp}$ of $E$. canis.

\section{Table 1}

The sequences of oligonucleotides used in the nested PCR of Ehrlichia canis detection in naturally infected dogs in Abeokuta, Nigeria

\begin{tabular}{lllc}
\hline Primer set & \multicolumn{1}{c}{ Primer sequence $\left(5^{\prime}-3^{\prime}\right)$} & Reference & $\begin{array}{c}\text { Band } \\
\text { size }\end{array}$ \\
\hline ECC & AGAACGAACGCTGGCGGCAAGC & & \\
ECB & CGTATTACCGCGGCT GCTGGCA & Murphy et al. (1998) & 478 \\
ECAN5 & CAATTATTTATAGCCTCTGGCTCTGGCTATAGG & & \\
HE3 & TATAGGT ACCGTCATTATCTTCCCTAT & Milanjeet et al. (2014) & 398 \\
\hline
\end{tabular}

Amplification was carried out in $20 \mu \mathrm{l}$ final volume containing $10 \mu \mathrm{l}$ of $2 \times$ mastermix (Syd Labs, Inc., USA), $8 \mu 1$ of nuclease-free water and $0.5 \mu 1$ (about $10 \mathrm{pmol}$ ) each of forward and reverse primer, and $1 \mu \mathrm{l}$ (about $50 \mathrm{ng}$ ) of the genomic DNA in a personal cycler (Biorad, USA). The cycling conditions for the primary PCR reactions were: $94{ }^{\circ} \mathrm{C}$ for 3 min initial denaturation, 30 cycles 
of $94{ }^{\circ} \mathrm{C}$ for $60 \mathrm{~s}, 59.5{ }^{\circ} \mathrm{C}$ for $60 \mathrm{~s}$ and $72{ }^{\circ} \mathrm{C}$ for $120 \mathrm{~s}$ with final extension at $72{ }^{\circ} \mathrm{C}$ for $8 \mathrm{~min}$. The nested PCR was carried out also in $20 \mu \mathrm{l}$ final volume containing $10 \mu 1$ of $2 \times$ mastermix (Syd Labs, Inc., USA), $8 \mu 1$ of nuclease-free water and $0.5 \mu 1$ (about $10 \mathrm{pmol}$ ) each of forward and reverse primer, and $1 \mu 1$ of the primary PCR product as the template. The PCR conditions were: initial denaturation at $94{ }^{\circ} \mathrm{C}$ for $3 \mathrm{~min}$, followed by two amplification steps: the first step consisted of three cycles of $94{ }^{\circ} \mathrm{C}$ for $1 \mathrm{~min}, 55^{\circ} \mathrm{C}$ for $2 \mathrm{~min}, 72^{\circ} \mathrm{C}$ for $1.5 \mathrm{~min}$, while the second step consisted of 37 cycles of $94^{\circ} \mathrm{C}$ for $1 \mathrm{~min}, 55^{\circ} \mathrm{C}$ for $2 \mathrm{~min}$, $72{ }^{\circ} \mathrm{C}$ for $1.5 \mathrm{~min}$ with final extension at $72{ }^{\circ} \mathrm{C}$ for $8 \mathrm{~min}$. In every amplification, a negative control was included using nuclease-free water instead of DNA.

The PCR products were visualised in $1.5 \%$ agarose gel that was stained with ethidium bromide (Amresco, USA) in $1 \times$ TAE buffer $(40 \mathrm{mM}$ Tris acetate and $1 \mathrm{mM}$ EDTA) and electrophoresed at $80 \mathrm{~V}$ for $60 \mathrm{~min}$ along with $10 \mu 1$ of 100 bp DNA ladder (BioExpress, Kaysville, UT, USA), and then visualised with UV transilluminator (Spectroline, USA). Gel picture was captured using a handheld camera (Samsung WB50F, China).

\section{Sequence and phylogenetic analyses}

To confirm the PCR assay results, four PCR products from the samples that showed expected band size were sequenced directly using Big Dye Terminator cycle sequencing kit (Applied Biosystems, Foster City, CA, USA) in the Core Lab of Cornell University Central Laboratory, USA using forward primer ECAN5. Search for homologous sequences in the GenBank was performed using BLASTn (www.ncbi.nlm.nih.gov/BLAST). The obtained sequences were viewed, aligned and compared using BioEdit ${ }^{\circledR}$ (version 7.0.9.0) software. The sequences were also aligned with other published $E$. canis $16 \mathrm{~S}$ rRNA sequences of dogs from around the world. Phylogenetic analysis of the sequences, including those obtained from GenBank were constructed using Maximum Composite Likelihood and Neighbour-Joining methods (Tamura et al., 2004) with a bootstrap confidence interval of 1000 replicates in Molecular Evolutionary Genetic Analysis (MEGA 5.0). The sequences obtained from this study have been deposited in GenBank under the accession number KY434110, KY434111, KY434112 and KY434113.

\section{Data analysis}

The data were summarised using descriptive statistics. The prevalences of E. canis obtained by PCR and microscopy as well as the prevalences within ages, sexes, and breeds of dogs were compared using Student's $t$-test. $\mathrm{P}<0.05$ was considered statistically significant. The analysis was carried out in SPSS version 19 software. 


\section{Ethical considerations}

Ethical approval (No. FUNAAB/COLVET/CREC/009/17) was obtained from the Ethical Committee of the College of Veterinary Medicine, Federal University of Agriculture Abeokuta, Nigeria, before commencing the project.

\section{Results}

Detection of $\mathrm{E}$. canis by microscopy and $P C R$

Out of the 205 dogs screened by microscopy, ehrlichial morulae were detected in the blood (two monocytes and one neutrophil) of three (1.5\%) dogs (Fig. 1). Gel electrophoresis of the nested PCR products revealed $47(22.9 \%)$ of the tested dogs to have a band size of about $398 \mathrm{bp}$ which corresponded to the expected fragment size of the amplified partial region of the 16S rRNA gene of E. canis. The three samples that were positive by microscopy were also positive by PCR. Among the screened dogs, there were more females (103) than males (102). The occurrence of $E$. canis in mongrels $(\mathrm{n}=2,6.5 \%)$, was significantly $(\mathrm{P}<0.05)$ lower compared to exotic breeds $(\mathrm{n}=45,25.9 \%)$, but the occurrence in female dogs $(23.3 \%)$ was not significantly different from that in males $(22.5 \%)\left(\mathrm{X}^{2}=0.02, \mathrm{P}=0.09\right)$. As regards age, the occurrence of $E$. canis in dogs less than 12 months old $(26 \%)$ was not significantly different from that in dogs older than one year $(21 \%)\left(\mathrm{X}^{2}=0.068, \mathrm{P}=0.4\right)$.
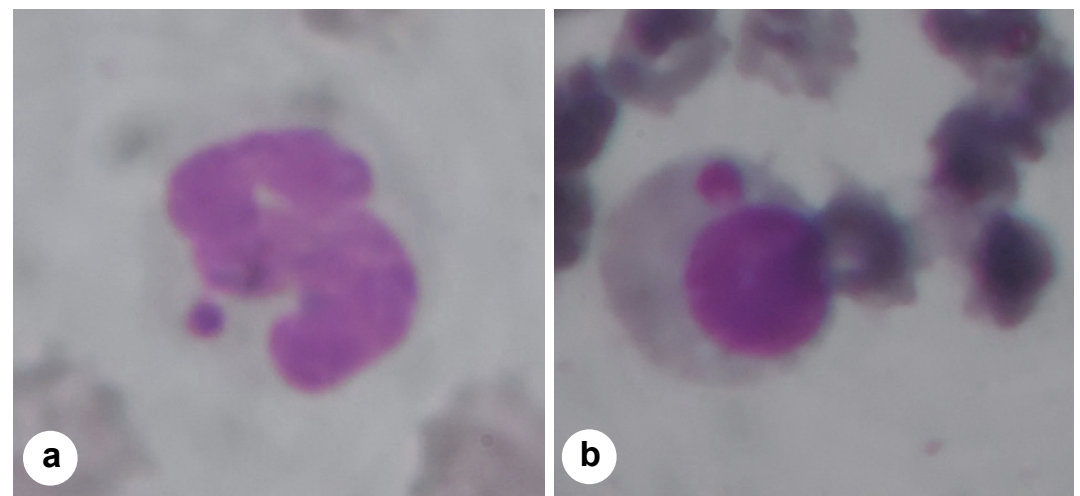

Fig. 1a-b. Microscopic screening of Ehrlichia canis in naturally infected dogs using a field-stained blood smear. a: Neutrophil infected by the morulae of E. canis; b: E. canis in an infected monocyte. Plates ( $a$ and $b$ ) were re-magnified at $\times 12$ of a Samsung (WB50F) camera

Sequences and phylogenetic analysis

The lengths of the sequences obtained from this study range from 374 to 376 bp with an average G-C content of 37\%. Homology search revealed that se- 
quences obtained from this study had $98-9 \%$ homology with the sequences deposited in GenBank (DQ379966, DQ228505, KP844663, KF878949, KX180945, KX898137, LC057655, M73226, NR118741, JX893522, U54805, KP642754, KJ995842, KJ995837 and KF972451). The aligned autochthonous sequences were less polymorphic with sequences KY434110 showing insertion (C) at point 994 and sequences KY434110 and KY434111 showing alterations $(\mathrm{A} \rightarrow \mathrm{C})$ at point 947 . When these sequences were aligned with those previously reported in Nigeria by Kamani et al. (2013), there were considerable variations characterised by complete deletion (at points 926, 962 and 1045), insertion (at points 886, 894, 944, 994 and 1024) and alterations at $912(\mathrm{~T} \rightarrow \mathrm{A}), 915(\mathrm{~A} \rightarrow \mathrm{C}), 919-921$ $(\mathrm{AGT} \rightarrow \mathrm{GCT})$ and $941-942(\mathrm{TA} \rightarrow \mathrm{AC})$.

The phylogenetic tree inferred by Maximum Likelihood and Neighbour Joining methods showed the same topologies (Figs 2 and 3). The trees clearly separated the sequences reported previously in Nigeria from the autochthonous sequences into two different clades. The sequences from this study clustered with those from India, Brazil and Angola, while those previously reported in Nigeria clustered among those from the USA, Turkey, Switzerland, Israel, Japan, South America, and Tahiti.

\section{Discussion}

This study attempted to shed more light on the occurrence of canine ehrlichiosis and the molecular characteristics of $E$. canis detected in naturally infected dogs in South West Nigeria by microscopy and by nested PCR that targeted a partial region of the $16 \mathrm{~S}$ rRNA gene.

The occurrence of ehrlichial morulae in $1.5 \%$ of the dogs sampled and screened by microscopy could not be compared due to the paucity of data and reports on microscopic detection of the parasite in Nigerian dogs. This may be an indication of how difficult microscopic detection of Ehrlichia parasites could be in the blood. The detection of morulae in both monocytes and granulocytes in this study may suggest that both granulocytic and monocytic ehrlichiosis exist among the dog population in Nigeria; hence, there is a need for a wide-scale molecular screening to ascertain which of the Ehrlichia species is/are available in Nigeria as those found in monocytes are generally believed to be either E. canis or E. chaffeensis and those found in granulocytes to be E. ewingii (Blanco and Oteo, 2002; Bowman et al., 2009). Although the infection stage was not classified in the dogs sampled in this study, the three cases in which morulae were detected by microscopy may represent acute infection as suggested by other workers (Aguero-Rosenfeld et al., 1996; Bakken et al., 1996) who posited that morulae are usually detected in blood smears only during acute febrile episodes.

The detection of E. canis DNA in $22.9 \%$ of the sampled population of dogs was higher than the prevalence of $12.7 \%$ reported by Kamani et al. (2013) 
in the northern part of Nigeria and those reported by Nazari et al. (2013) and Lasta et al. (2013) in Malaysia and Brazil, respectively. The higher occurrence reported in this study may partly be associated with the predominance of the tick vector (Rhipicephalus sanguineus) of the parasite in the study area (Oke et al., 2013) and may be due to optimum environmental conditions that favour its breeding. Since we only amplified with $E$. canis specific primers, there is a need for further studies including other species-specific primers to determine whether mixed infections of these Ehrlichia parasites are possible or exist.

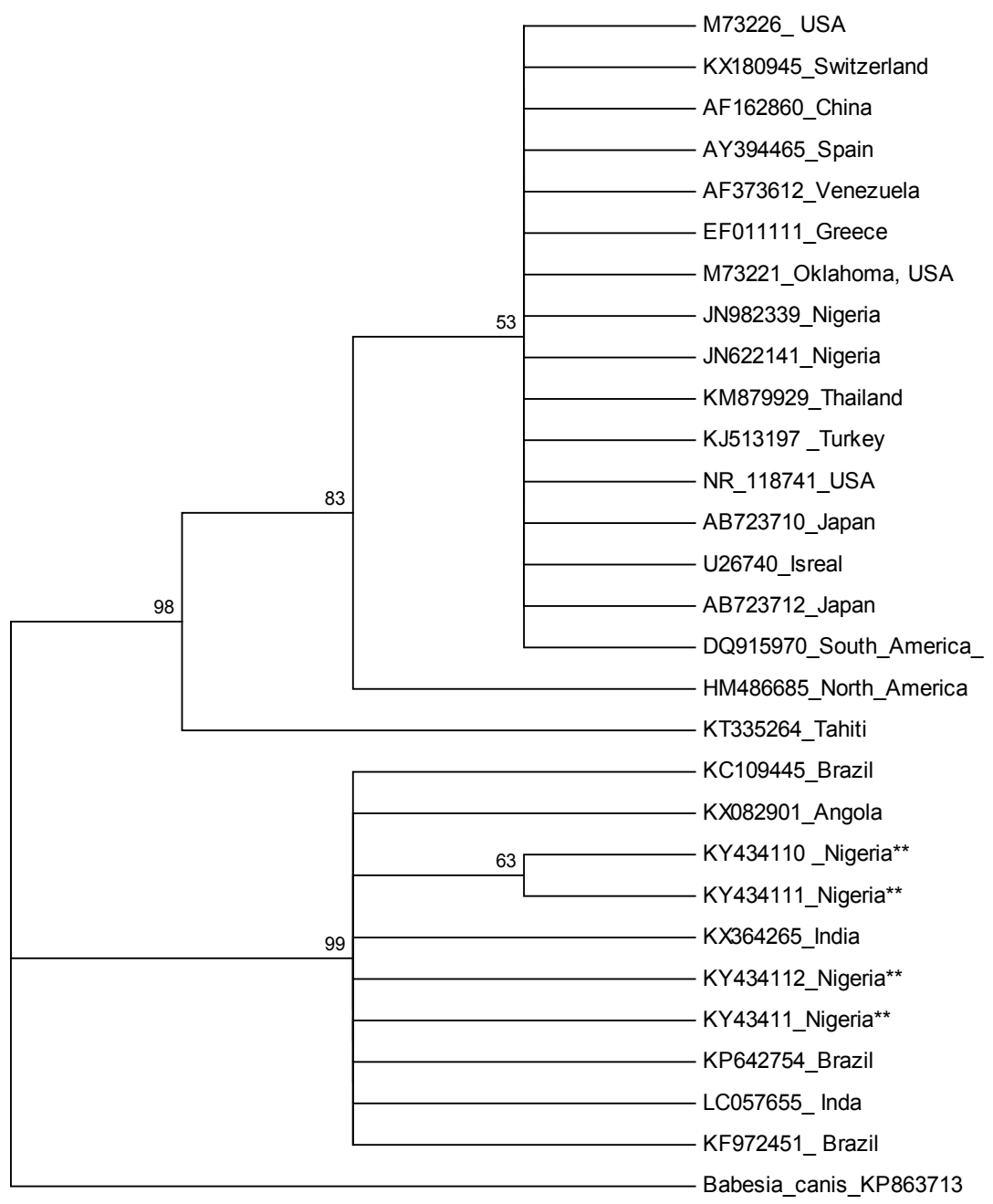

Fig. 2. Phylogenetic analysis of $16 \mathrm{~S}$ rRNA gene partial sequences of $E$. canis isolates inferred using the Maximum Likelihood method. The percentage (50\% and above) of replicate trees in which the associated taxa clustered together in the bootstrap test (1000 replicates) are shown next to the branches 


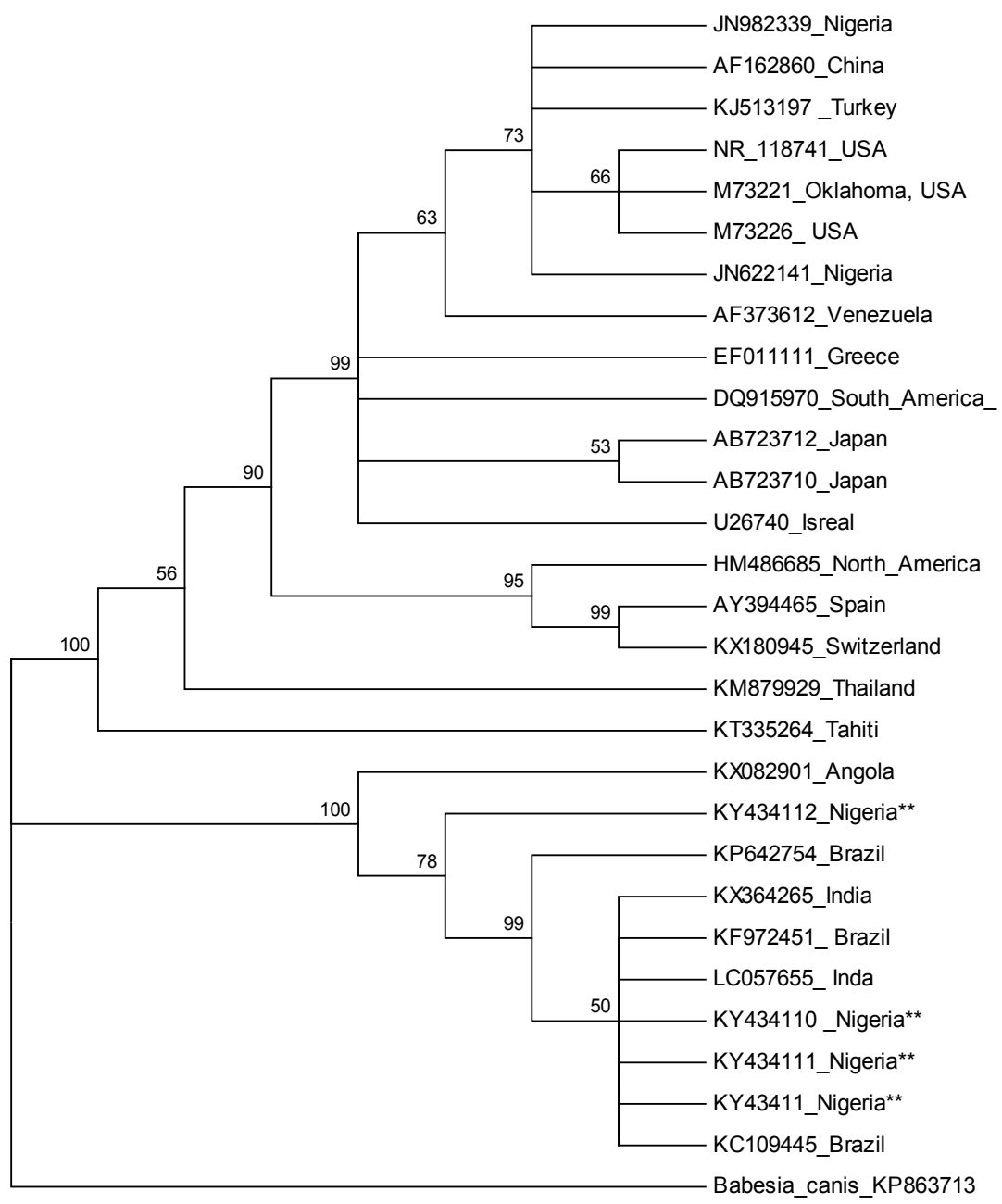

Fig. 3. Phylogenetic analysis of $16 \mathrm{~S}$ rRNA gene partial sequences of E. canis isolates inferred using the Neighbour-Joining method. The percentage (50\% and above) of replicate trees in which the associated taxa clustered together in the bootstrap test (1000 replicates) are shown next to the branches

The E. canis sequences that were used to construct the phylogenetic trees exhibit diphyletic clades, separating the autochthonous sequences from those previously described in Nigeria. The separation may be an indication that more than one strain of E. canis are in circulation among the dog population in Nigeria. Clinical manifestation was not reported either in our study or in the previous study on ehrlichial infection of dogs in Nigeria; hence, further investigations on the pathogenicity of these strains in infected dogs are needed. The diphyletic clades exhibited by the autochthonous 16S rRNA partial sequences and those re- 
ported previously in Nigeria are not supported by some of the previous works on the phylogenetics of E. canis (Vinasco et al., 2007; Vargas-Hernández et al., 2012). This could be an indication that the 16S rRNA gene of E. canis strains in Nigeria is more polymorphic than those reported elsewhere and this may have potential implications on the clinical outcome and management of canine ehrlichiosis in Nigeria. Generally, E. canis (Dawson et al., 1993; Unver et al., 2001; Perez et al., 2006), E. chaffeensis (Dawson et al., 1993; Chen et al., 1997; Rojas et al., 2015) and E. ewingii (Buller et al., 1999; Allen et al., 2014) have been reported in human ehrlichiosis around the world but not in Nigeria. This does not necessarily preclude the presence of ehrlichial infection in humans in Nigeria and, as such, the screening for such parasites should be considered in human febrile conditions.

In conclusion, this study provides molecular evidence that shows the presence of an E. canis strain in naturally infected dogs in Abeokuta (South West Nigeria), which is genetically different from the strain previously reported in the northern part of Nigeria and more closely related to sequences from Brazil and India.

\section{References}

Aguero-Rosenfeld, M. E., Horowitz, H. W. and Wormser, G. P. (1996): Human granulocytic ehrlichiosis: a case series from a medical center in New York State. Ann. Intern. Med. 125, 904-908.

Aguiar, D. M., Hagiwara, M. K. and Labruna, M. B. (2008): In vitro isolation and molecular characterization of an Ehrlichia canis strain from São Paulo, Brazil. Braz. J. Microbiol. 39, 489-493.

Allen, M. B., Pritt, B. S., Sloan, L. M., Paddock, C. D., Musham, C. K., Ramos, J. M., Cetin, N. and Rosenbaum, E. R. (2014): First reported case of Ehrlichia ewingii involving human bone marrow. J. Clin. Microbiol. 52, 4102-4104.

Bakken, J. S., Krueth, J., Wilson-Nordskog, C., Tilden, R. L., Asanovich, K. and Dumler, J. S. (1996): Clinical and laboratory characteristics of human granulocytic ehrlichiosis. J. Am. Med. Assoc. 275, 199-205.

Blanco, J. R. and Oteo, J. A. (2002): Human granulocytic ehrlichiosis in Europe. Clin. Microbiol. Infect. 8, 763-772.

Bowman, D., Little, S. E., Lorentzen, L., Shields, J., Sullivan, M. P. and Carlin, E. P. (2009): Prevalence and geographic distribution of Dirofilaria immitis, Borrelia burgdorferi, Ehrlichia canis, and Anaplasma phagocytophilum in dogs in the United States: results of a national clinic-based serologic survey. Vet. Parasitol. 160, 138-148.

Buller, R. S., Arens, M., Hmiel, S. P., Paddock, C. D., Sumner, J. W., Rikihisa, Y., GaudreaultKeener, M., Manian, F. A., Liddell, A. M., Schmulewitz, N. and Storch, G. A. (1999): Ehrlichia ewingii, a newly recognized agent of human ehrlichiosis. N. Engl. J. Med. 341, 148-155.

Cardoso, L., Oliveira, A. C., Granada, S., Nachum-Biala, Y., Gilad, M., Lopes, A. P., Sousa, S. R., Vilhena, H. and Baneth, G. (2016): Molecular investigation of tick-borne pathogens in dogs from Luanda, Angola. Parasit. Vectors 9, 252.

Chen, S. M., Yu, X. J., Popov, V. L., Westerman, E. L., Hamilton, F. G. and Walker, D. H. (1997): Genetic and antigenic diversity of Ehrlichia chaffeensis: comparative analysis of a novel human strain from Oklahoma and previously isolated strains. J. Infect. Dis. 175, 856-863. 
Cicuttin, G. L., De Salvo, M. N. and Gury Dohmen, F. E. (2016): Molecular characterization of Ehrlichia canis infecting dogs, Buenos Aires. Ticks Tick Borne Dis. 7, 954-957.

Dagnone, A. S., Souza, A. I. D., André, M. R. and Machado, R. Z. (2009): Molecular diagnosis of Anaplasmataceae organisms in dogs with clinical and microscopical signs of ehrlichiosis. Rev. Bras. Parasitol. Vet. 18, 20-25.

Dawson, J. E., Candal, F. J., George, V. G. and Ades, E. W. (1993): Human endothelial cells as an alternative to DH82 cells for isolation of Ehrlichia chaffeensis, E. canis, and Rickettsia rickettsii. Pathobiology 61, 293-296.

Dumler, J. S., Choi, K., Garcia-Garcia, J. C., Barat, N. S., Scorpio, D. G., Garyu, J. W., Grab, D. J. and Bakken, J. S. (2005): Human granulocytic anaplasmosis and Anaplasma phagocytophilum. Emerg. Infect. Dis. 11, 1828-1834.

Engvall, E. O., Pettersson, B., Persson, M., Artursson, K. and Johansson, K. E. (1996): A 16S rRNA-based PCR assay for detection and identification of granulocytic Ehrlichia species in dogs, horses, and cattle. J. Clin. Microbiol. 34, 2170-2174.

Fourie, J. J., Stanneck, D., Luus, H. G., Beugnet, F., Wijnveld, M. and Jongejan, F. (2013): Transmission of Ehrlichia canis by Rhipicephalus sanguineus ticks feeding on dogs and on artificial membranes. Vet. Parasitol. 197, 595-603.

Harrus, S., Perlman-Avrahami, A., Mumcuoglu, K. Y., Morick, D., Eyal, O. and Baneth, G. (2011): Molecular detection of Ehrlichia canis, Anaplasma bovis, Anaplasma platys, Candidatus midichloria mitochondrii and Babesia canis vogeli in ticks from Israel. Clin. Microbiol. Infect. 17, 459-463.

Kaewmongkol, G., Maneesaay, P., Suwanna, N., Tiraphut, B., Krajarngjang, T., Chouybumrung, A., Kaewmongkol, S., Sirinarumitr, T., Jittapalapong, S. and Fenwick, S. (2016): First detection of Ehrlichia canis in cerebrospinal fluid from a non-thrombocytopenic dog with meningoencephalitis by broad-range PCR. J. Vet. Intern. Med. 30, 255-259.

Kamani, J., Baneth, G., Mumcuoglu, K. Y., Waziri, N. E., Eyal, O., Guthmann, Y. and Harrus, S. (2013): Molecular detection and characterization of tick-borne pathogens in dogs and ticks from Nigeria. PLoS Negl. Trop. Dis. 7, e2108.

Lasta, C. S., Santos, A. P., Messick, J. B., Oliveira, S. T., Biondo, A. W., Vieira, R. F. C., Dalmolin, M. L. and González, F. H. D. (2013): Molecular detection of Ehrlichia canis and Anaplasma platys in dogs in Southern Brazil. Rev. Bras. Parasitol. Vet. 22, 360-366.

Lauzi, S., Maia, J. P., Epis, S., Marcos, R., Pereira, C., Luzzago, C., Santos, M., Puente-Payo, P., Giordano, A. and Pajoro, M. (2016): Molecular detection of Anaplasma platys, Ehrlichia canis, Hepatozoon canis and Rickettsia monacensis in dogs from Maio Island of Cape Verde archipelago. Ticks Tick Borne Dis. 7, 964-969.

Liddell, A. M., Stockham, S. L. and Scott, M. A. (2003): Predominance of Ehrlichia ewingii in Missouri dogs. J. Clin. Microbiol. 41, 4617-4622.

McBride, J. W., Corstvet, R. E., Gaunt, S. D., Chinsangaram, J., Akita, G. Y. and Osburn, B. I. (1996): PCR detection of acute Ehrlichia canis infection in dogs. J. Vet. Diagn. Invest. 8, 441-447.

Milanjeet, H. S., Singh, N., Singh, N., Singh, C. and Rath, S. (2014): Molecular prevalence and risk factors for the occurrence of canine monocytic ehrlichiosis. Vet. Med. 59, 129-136.

Nazari, M., Lim, S. Y., Watanabe, M., Sharma, R. S. K., Cheng, N. A. B. Y. and Watanabe, M. (2013): Molecular detection of Ehrlichia canis in dogs in Malaysia. PLoS Negl. Trop. Dis. 7, e1982.

Oke, O. A., Idowu, A. B., Ademolu, K. O. and Bamgbose, O. J. (2013): Prevalence of tick infestation on dogs (Canis spp.) presented at the Veterinary Teaching Hospital, Federal University of Agriculture Abeokuta, South West Nigeria. Adv. Sci. Tech. 7, 20-24.

Perez, M., Bodor, M., Zhang, C., Xiong, Q. and Rikihisa, Y. (2006): Human infection with Ehrlichia canis accompanied by clinical signs in Venezuela. Ann. N. Y. Acad. Sci. 1078, 110-117.

Rojas, N., Castillo, D. and Marin, P. (2015): Molecular detection of Ehrlichia chaffeensis in Humans, Costa Rica. Emerg. Infect. Dis. 21, 532-534. 
Souza, B. M. P. S., Leal, D. C., Barboza, D. C. P. M., Uzêda, R. S., Alcântara, A. C., Ferreira, F., Labruna, B. M., Gondim, L. F. P. and Franke, C. R. (2010): Prevalence of ehrlichial infection among dogs and ticks in Northeastern Brazil. Rev. Bras. Parasitol. Vet. Jaboticabal. 19, 89-93.

Suksawat, J., Xuejie, Y., Hancock, S. I., Hegarty, B. C., Nilkumhang, P. and Breitschwerdt, E. B. (2001): Serologic and molecular evidence of coinfection with multiple vector-borne pathogens in dogs from Thailand. J. Vet. Intern. Med. 15, 453-462.

Takeet, M. I., Fagbemi, B. O., Donato, M. D., Yakubu, A., Rodulfo, H. E., Peters, S. O., Wheto, M. and Imumorin, I. G. (2013): Molecular survey of pathogenic trypanosomes in naturally infected Nigerian cattle. Res. Vet. Sci. 94, 555-561.

Tamura, K., Nei, M. and Kumar, S. (2004): Prospects for inferring very large phylogenies by using the neighbor-joining method. Proc. Natl Acad. Sci. U.S.A. 101, 11030-11035.

Unver, A., Perez, M., Orellana, N., Huang, H. and Rikihisa, Y. (2001): Molecular and antigenic comparison of Ehrlichia canis isolates from dogs, ticks, and a human in Venezuela. J. Clin. Microbiol. 39, 2788-2793.

Vargas-Hernández, G., André, M. R., Faria, J. L. M., Munhoz, T. D., Hernandez-Rodriguez, M., Machado, R. Z. and Tinucci-Costa, M. (2012): Molecular and serological detection of Ehrlichia canis and Babesia vogeli in dogs in Colombia. Vet. Parasitol. 186, 254-260.

Vinasco, J., Li, O., Alvarado, A., Diaz, D., Hoyos, L., Tabachi, L. and Moro, M. H. (2007): Molecular evidence of a new strain of Ehrlichia canis from South America. J. Clin. Microbiol. 45, 2716-2719. 